\title{
SISTEMA EMBARCADO PARA O MONITORAMENTO DE VACINAS: APRENDIZAGEM BASEADA EM PROJETOS NO PET EMB
}

DOI: 10.37702/2175-957X.COBENGE.2021.3431

Thiago Almeida Eidt - thiagoeidt@hotmail.com

Universidade Federal de Santa Catarina

Rua Presidente Prudente de Moraes 235

89218-000 - Joinville - SC

Camila Rossignatti - camilarossignatti@hotmail.com

Universidade Federal de Santa Catarina

Rua Alberto Santos Dumont 1296

13334-150 - Indaiatuba - SP

Guilherme Cervi Garcia - guilhermecervigarcia@gmail.com

Universidade Federal de Santa Catarina

Romilson França Filho - romilsonffilho@gmail.com

Universidade Federal de Santa Catarina

Rua João chefe dippe 143

- Joinville - SC

Carlos Mauricio Sacchelli - carlos.sacchelli@gmail.com

Universidade Federal de Santa Catarina

dr. joão colin 2700

89218-350 - joinville - SC

João Carlos Xavier - j.xavier@ufsc.br

Universidade Federal de Santa Catarina

Felix Heinzelmann 961

89218-321 - Joinville - SC

Juan Camilo Castellanos Rodriguez - juanc@utfpr.edu.br Universidade Tecnologica Federal do Paraná

Av. Sete de Setembro 3165

80230-901 - Curitiba - PR 
Resumo: $O$ presente trabalho apresenta o relato de experiência dos estudantes do Programa de Educação Tutorial do Centro de Engenharias da Mobilidade da UFSC durante a pandemia de COVID-19. Os estudantes desenvolveram um dispositivo de baixo custo para monitoramento de temperatura de geladeiras domésticas utilizadas no armazenamento de vacinas. A metodologia adotada na execução das atividades foi a metodologia baseada em projetos, sendo o resultado final do processo a aquisição de habilidades de trabalho em equipe na modalidade remota e conhecimentos em sistemas embarcados de baixo custo. Além disso, também foi realizada a construção de um protótipo de sistema embarcado formado por um aplicativo para dispositivos móveis, que emite avisos em caso de falta de energia e temperaturas próximas ou fora da faixa desejada, e uma parte de hardware, que adquire e envia os dados de temperatura via internet, para uma planilha do Google Sheets ${ }^{T M}$.

Palavras-chave: Vacinas. Monitoramento. Mobile. 


\section{INTRODUÇÃO}

No ano de 2019 o mundo foi impactado pela pandemia causada pelo novo coronavírus, o vírus SARS-CoV-2, responsável pela COVID-19, que já infectou cerca de 128 milhões de pessoas mundialmente, sendo 97,194 milhões de brasileiros, com mais de 2,81 milhões de óbitos, do quais 321.780 mil são do Brasil, desde seu início, em dezembro de 2019, até abril de 2021 (W. H. Organization, 2021). L. Mikhailov et al. (2020) expõe que o vírus se propaga por meio do contato direto ou próximo de pessoas contaminadas através de secreções ou gotículas nasal ou bucal, ou pelo contato indireto com objetos contaminados. Os sintomas comuns causado pelo SARS-CoV-2 são tosse seca, febre e cansaço, podendo se agravar causando dor no peito, perda de fala ou movimento e dificultando na respiração do infectado (W. H. Organization, 2020).

As vacinas, por sua vez, são as principais responsáveis por combater várias doenças, entre elas as virais, em vários países. Antes do seu surgimento, milhares de pessoas eram levadas a óbito devido doenças que foram erradicadas com o tempo. Um exemplo disso são doenças como a tuberculose e a varíola, sendo que esta última matou cerca de 300 milhões de pessoas entre 1896 e 1970. Em 1989, no Brasil, o uso das vacinas erradicou a poliomielite, doença que atingiu milhares de crianças, causando a paralisia infantil (Fiocruz, 2019). Rocha (2019) afirma que o Brasil é um dos países que mais oferece vacinas à população, disponibilizando no total 300 milhões de doses anuais. Diversas empresas como a Sinovac, Pfizer, Oxford, entre outras, começaram a pesquisar e produzir vacinas contra o COVID-19, sendo algumas já regulamentadas pela Agência Nacional de Vigilância Sanitária, Anvisa, possibilitando a distribuição das mesmas ao Programa Nacional de Imunização (PNI). Com as vacinas desenvolvidas foi possível dar início à vacinação de milhões de cidadãos ao redor do mundo.

Vacinas são materiais termolábeis que sofrem alterações na sua composição se não forem mantidas em temperaturas específicas, geralmente entre +2 e +8 graus Celsius (Ministério da Saúde, 2019). Para que mantenham sua vida útil e alcancem seu objetivo, que é proteger o indivíduo vacinado, é preciso mantê-las dentro dessa faixa de temperatura. Em todo município brasileiro as vacinas são armazenadas e distribuídas pelo Sistema Único de Saúde, o SUS.

A estrutura hierárquica de distribuição de vacinas e as boas práticas para seu armazenamento podem ser encontradas no manual distribuído pelo Ministério da Saúde, chamado Manual de Rede de Frio (Ministério da Saúde, 2019). Chama-se Rede de Frio o processo logístico e hierárquico envolvido desde a produção das vacinas até sua administração à população pelos agentes de saúde. O Manual de Rede de Frio também contempla as orientações adequadas para o correto armazenamento das vacinas que serão disponibilizadas à população (Ministério da Saúde, 2019).

No Brasil, as vacinas estão disponíveis à população nas Unidades Básicas de Saúde (UBS), que as armazenam nas salas de vacina. Nesse ambiente todas as vacinas devem ser mantidas entre +2 graus Celsius e +8 graus Celsius.

Para o controle de armazenamento das vacinas nas UBSs há no mercado câmaras de vacina que monitoram sua temperatura interna, avisam sobre quedas de tensão na rede elétrica local e contam com dispositivos que a mantém funcionando por até vinte e quatro horas no caso de falta de energia (Indrel, 2019). No entanto, seu custo é elevado, em torno de 13.500,00 em 2016 (Tunápolis, 2019), dificultando a aquisição dessas câmaras por parte das unidades dos municípios com pouco orçamento. Nesse caso, o Manual de Rede de Frios sugere a utilização de geladeiras domésticas com capacidade mínima de 280 litros, com o monitoramento da temperatura sendo feito utilizando termômetros de máxima e mínima. As temperaturas internas e externas das geladeiras devem ser anotadas pelo 
menos duas vezes ao dia em formulário específico, uma no início da jornada de trabalho e outra ao final.

Ministério da Saúde e Felizona (2019) dizem que com o uso de uma geladeira doméstica e a forma de controle adotada, oscilações de temperatura que ocorram durante o dia ou um problema na unidade de armazenamento de vacinas, (e.g. a queda de tensão da rede local), podem gerar variações na sua temperatura além dos valores permitidos. Isto compromete a efetividade do material armazenado sem que os responsáveis possam intervir antes que isso ocorra. As vacinas comprometidas devem ser testadas para comprovar a sua eficiência ou descartadas. Porém, essas duas alternativas geram um custo extra para o município e consequentemente um gasto desnecessário de dinheiro público. Usando como exemplo o município de Petrolina, em Pernambuco, no ano de 2013, os fatores determinantes das perdas das vacinas no município foram a falta de energia elétrica, com $43,75 \%$, seguida de falha no equipamento de refrigeração, com $33,33 \%$, ambas as situações gerando perdas através da exposição dos imunobiológicos a temperaturas inadequadas. Tais falhas ocasionaram um prejuízo de $\mathrm{R} \$ 72.864,23$ aos cofres públicos do município (Da Silva Santos JA, et al, 2016).

O Programa de Educação Tutorial de Engenharias da Mobilidade (PET EMB) é um grupo composto por um tutor e alunos de engenharia da Universidade Federal de Santa Catarina (UFSC). O programa visa proporcionar a vivência em atividades acadêmicas que integram o ensino, pesquisa e extensão. As atividades extracurriculares realizadas pelos integrantes complementam a experiência e a formação dos mesmos, preparando-os para o mercado de trabalho e a superar as dificuldades e desafios que podem surgir durante suas vidas profissionais (PET EMB, 2021).

Assim, considerando o papel do PET EMB na formação dos estudantes de engenharia e a necessidade de um dispositivo que permita um monitoramento automatizado da temperatura de geladeiras utilizadas no armazenamento de vacinas, foi proposto a construção de um dispositivo de Monitoramento de Temperatura, interativo, por meio de um aplicativo para celular, confiável e de baixo custo para as salas de vacina do país. Com o equipamento desenvolvido pretende-se evitar perdas de vacinas devido sua exposição a temperaturas inadequadas, ocasionadas, por exemplo, por quedas de energia, e o acompanhamento da temperatura das geladeiras de forma remota. Além do mais, com a chegada das novas vacinas contra o COVID-19, ainda escassas no mercado, tal monitoramento e a preservação das vacinas nas temperaturas adequadas torna-se ainda mais importante. O grupo à frente do projeto é composto por alunos do PET EMB da UFSC - campus Joinville e dois professores/coordenadores. A metodologia adotada para 0 desenvolvimento do projeto foi a aprendizagem baseada em projetos, em que os alunos se envolvem com tarefas e desafios para desenvolver um projeto ou um produto, integrando seus conhecimentos e estimulando o desenvolvimento de competências, como trabalho em equipe, protagonismo e pensamento crítico, levando a um processo de aprendizagem ativa (Porvir, 2021).

Desse modo, o objetivo principal desse trabalho é apresentar uma solução para o monitoramento da temperatura de geladeiras utilizadas no armazenamento de vacinas na rede pública de saúde a partir de uma metodologia de aprendizado por projetos.

O artigo está divido em três seções, que abordarão a descrição da metodologia adotada para o desenvolvimento do projeto, a criação do hardware e software e os resultados obtidos e, por fim, as conclusões. 


\section{METODOLOGIA}

A condução proposta para o desenvolvimento do projeto é identificada como Aprendizagem Baseada em Projetos, termo original Project Based Learning (PBL), que tem como objetivo a aprendizagem ativa e em grupo. Assim, o foco está na construção de conhecimento através da interação social e na aprendizagem autodirigida com monitoramento e suporte dos professores. A metodologia tem como base de ação a análise do problema alvo; período de aquisição de competências e ferramentas; a execução prática do projeto; e a avaliação em relação aos objetivos finais (Fonseca e Mattar, 2017). A figura abaixo apresenta as fases da Aprendizagem Baseada em Projetos (Fonseca e Mattar, 2017) que foram adotadas no desenvolvimento do dispositivo de monitoramento de temperatura para geladeiras utilizadas para armazenamento de vacinas:

Figura 1 - Etapas da Aprendizagem Baseada em Projetos

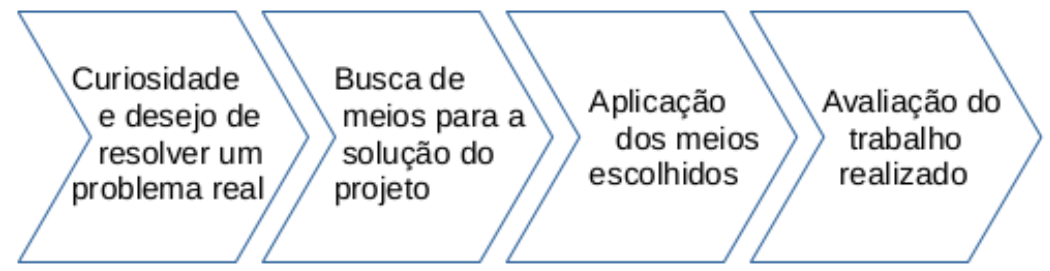

Fonte: Os autores

Na primeira fase (curiosidade e desejo de resolver um problema real) os estudantes delimitam o tema que será abordado, o objetivo a ser alcançado com o projeto e o produto final a ser desenvolvido. A segunda fase (busca de meios para a solução do projeto) envolve a preparação para o desenvolvimento do projeto, organização das rotinas de estudo e trabalho, formação de grupos e a busca das informações. Na terceira fase (aplicação dos meios escolhidos) está contemplada a execução dos meios de trabalho e dos conhecimentos e a apresentação ao grupo das informações adquiridas. Por fim, a quarta fase (avaliação do trabalho realizado) engloba a apresentação do projeto e a avaliação do resultado final, tanto em relação ao produto final como em relação ao aprendizado dos estudantes.

Na sequência são descritas detalhadamente como ocorreram as fases citadas acima no trabalho aqui apresentado.

\subsection{Curiosidade e desejo de resolver um problema real}

O tema escolhido como norteador do projeto, o monitoramento de temperatura de geladeiras utilizadas para armazenamento de vacinas, é proveniente do relato de profissionais de saúde em relação às limitações das ferramentas atualmente disponíveis para monitoramento e acompanhamento da temperatura dos equipamentos. Além disso, 0 contexto da pandemia de COVID-19 e o iminente lançamento das vacinas também foi levado em consideração na escolha do tema. Com a escassez de vacinas no mercado mundial, foi pensado em um tema que pudesse auxiliar no processo de armazenamento dessas vacinas dentro da faixa de temperaturas desejada, evitando perdas consideradas evitáveis, como por exemplo, por quedas de energia.

A partir da definição do tema, o projeto foi norteado por alguns dos requisitos que os profissionais de saúde elencaram, em entrevista, como sendo fundamentais para um dispositivo de monitoramento de temperatura:

- Monitorar a temperatura de uma geladeira doméstica; 
- Enviar avisos sobre faltas de energia;

- Permitir o monitoramento da temperatura de forma remota;

- Fornecer relatórios com o histórico de temperatura do equipamento;

- Não ser necessário realizar periodicamente trocas de bateria.

A partir desses requisitos deu-se início às pesquisas sobre regulamentações e protocolos referentes ao armazenamento de vacinas (tipo de refrigeradores permitidos, faixa de temperatura exigida, etc.).

Como resultado das pesquisas, constatou-se que o tipo de refrigerador mais utilizado para essa finalidade são refrigeradores convencionais/domésticos, os quais não possuem nenhum sistema dedicado para monitoramento de sua temperatura. Sendo assim, a concepção do projeto ficou baseada em monitorar a temperatura e enviar avisos sobre possíveis falhas como falta de energia, temperaturas próximas ao limite desejado e monitoramento da abertura/fechamento de porta (uma porta esquecida aberta por muito tempo pode ocasionar mudança no desempenho do equipamento e assim expor os imunobiológicos a temperaturas inadequadas). Realizou-se então a segunda etapa da pesquisa, a fim de reunir informações sobre produtos com essas características e que estão disponíveis no mercado. Com base nas pesquisas, a meta final estabelecida foi a construção de um equipamento que monitore a temperatura de uma geladeira doméstica e informe ao usuário, através de um aplicativo para dispositivos mobile, anomalias na temperatura e eventuais faltas de energia, a fim de evitar a exposição das vacinas a temperaturas inadequadas.

\subsection{Busca de meio para a solução do projeto}

Com base nas pesquisas realizadas, foi definido como meta a construção de um equipamento de baixo custo que monitore a temperatura de uma geladeira doméstica e informe ao usuário, através de um aplicativo para dispositivos mobile, anomalias na temperatura e eventuais faltas de energia.

Para que a meta fosse alcançada e o produto final desenvolvido, o projeto foi fragmentado em duas linhas de ação: uma chamada hardware e outra software. Assim, houve a divisão da equipe de quatro estudantes em dois grupos iguais. A ideia da separação em grupos foi direcionar seus integrantes para a aquisição das habilidades específicas de cada uma das áreas, as quais foram adquiridas conforme o projeto se desenvolveu.

Grupo da linha de software: os alunos alocados no desenvolvimento do software buscaram aprimoramento em programação, tendo como foco o desenvolvimento de aplicativos para dispositivos móveis, especificamente através do Android Studio.

Para o armazenamento dos dados coletados com os sensores de temperaturas e do status da porta do refrigerador os integrantes do grupo de software dedicaram sua atenção ao Google Sheets. Além disso, também buscaram conhecimentos sobre a plataforma Google Apps Script, a qual permite a automação do envio de dados à planilha do Google Sheets. Os resultados alcançados foram disponibilizados no serviço de armazenamento Google Drive, permitindo o acesso simultâneo a todos os integrantes do projeto.

Ficou definido também que os dados coletados pelo sensor de temperatura seriam armazenados no Google Sheets e o aplicativo faria a leitura dessas informações e compararia com a faixa de temperatura permitida, sendo essa de +2 até +8 graus Celsius. Caso o aplicativo detecte uma temperatura próxima ou fora do parâmetro estabelecido, o aplicativo deveria notificar o usuário. O aplicativo também deve notificar o usuário quando o sensor de temperatura não enviar os dados para serem armazenados, o que pode indicar falta de energia. 
Grupo da linha de hardware: Os estudantes do grupo de hardware buscaram conhecimento na área de programação de microcontroladores. Para a construção do produto final escolheu-se o microcontrolador ESP32 e sua placa de prototipagem NodeMCU-32. Este microcontrolador possui módulos como WiFi (que é o mecanismo principal de envio de dados coletados) e Bluetooth. Além disso, pode atuar com baixo consumo de energia e apresenta um elevado custo benefício.

Para o desenvolvimento da parte de hardware buscou-se inicialmente uma IDE (Integrated Development Environment) mais adequada para realizar a programação e o envio do programa para o microcontrolador. Foi selecionado o Arduino IDE, o qual tem interface com o ESP32 e funções predefinas em sua biblioteca.

Em relação aos periféricos utilizados para captação de dados como temperatura, abertura de porta e emissão de sinal sonoro em caso de porta aberta, fez-se a escolha, respectivamente, por: dois sensores de temperatura encapsulados (modelo DS18B20), um para o monitoramento da temperatura no interior do refrigerador e outro para o ambiente externo; um sensor fim de curso para detecção da abertura e fechamento de porta; um Buzzer ativo para emitir um sinal sonoro quando for detectado um período de porta aberta maior que o permitido.

Para a energização do sistema de hardware, o qual requer 3V, optou-se por um sistema de alimentação recarregável (para o caso de ocorrer uma queda de energia no local monitorado e evitar perdas de dados). Esse sistema utiliza uma bateria de lítio e um modulo TP4056, o qual está conectado através de um cabo mini USB a uma fonte de alimentação bivolt (220/110 V).

A composição da proposta de produto final pode ser verificada na representação esquemática mostrada na Figura 2.

Figura 2 - Representação da composição Hardware e Software:

(1) Microcontrolador; (2) Sistema de alimentação recarregável;

(3) Periféricos; (4) Alarme sonoro;(5) Comunicação; (6) Aplicativo

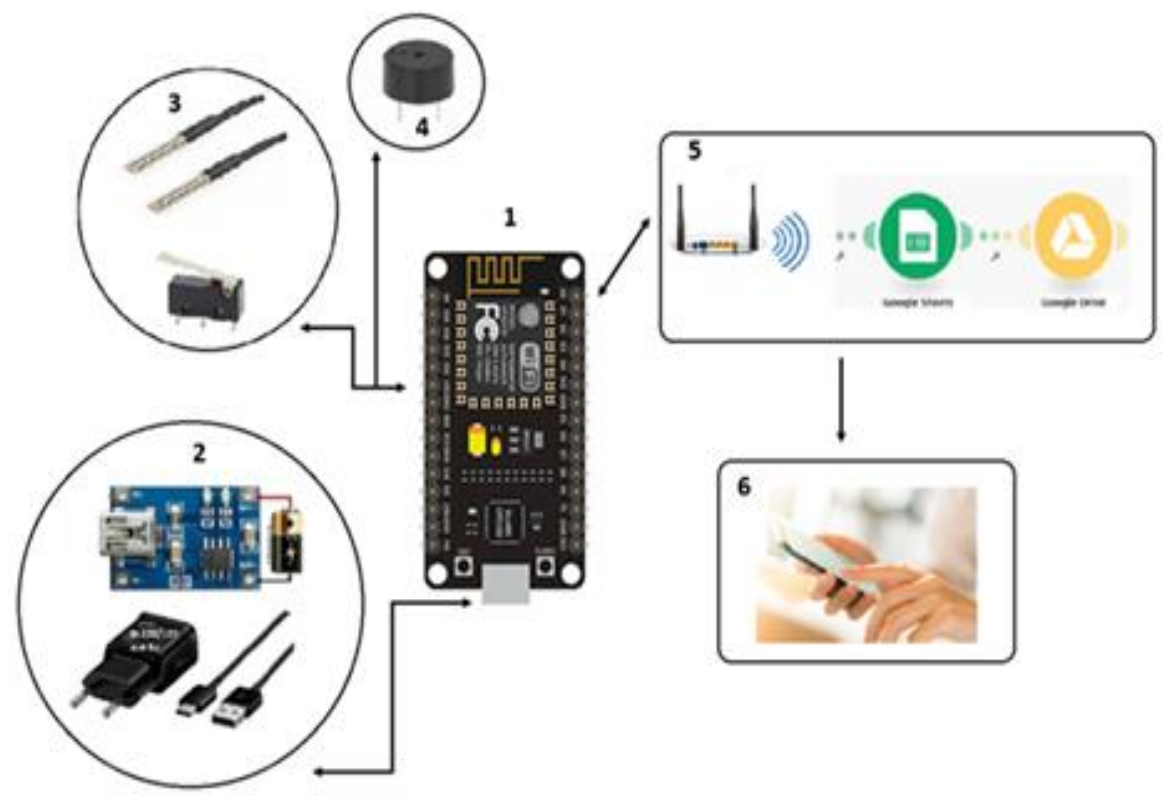

Fonte: Os Autores.

Devido ao momento de atividades presenciais restritas e a limitada acessibilidade a recursos, foi necessária a adequação da infraestrutura particular de cada integrante do projeto para a realização das atividades. Vale ressaltar que todas as atividades do projeto 
foram desenvolvidas em suas próprias casas, sem nenhum encontro presencial. Em especial em relação ao grupo de hardware, os componentes necessários para o desenvolvimento dessa parte do projeto foram comprados pela internet e entregues diretamente na casa dos estudantes.

As reuniões remotas substituíram as presenciais e foram realizadas através do Google Meet. Elas ocorreram semanalmente em um horário onde havia disponibilidade de todos os integrantes. Para o gerenciamento de informações como arquivos, vídeos e discussões dirigidas foi utilizada a plataforma Microsoft Teams.

A gestão de tempo e tarefas a serem realizadas foram organizadas através de um Diagrama de Gantt (Gantt chart), que descreve as metas a serem atingidas em cada semana e a definição/direcionamento das tarefas para as semanas seguintes.

Para o desenvolvimento das atividades de programação, tanto da parte de software como da parte de hardware, foram realizadas pesquisas na internet. Durante essas pesquisas buscou-se por soluções já existentes para problemas parecidos aos encontrados no desenvolvimento do projeto. Nas reuniões semanais os professores orientadores/coordenadores também forneciam dicas e orientações em relação à busca por soluções.

\subsection{Aplicação dos meios escolhidos}

Os resultados de cada semana de trabalho eram apresentados nas reuniões semanais por cada grupo. Nesses encontros cada estudante fazia observações sobre as tarefas que desenvolveu, as tarefas que estavam para desenvolver e os desafios encontrados na semana que passou.

Ficou ainda definido como produto final da equipe de software confeccionar um aplicativo para dispositivo mobile capaz de realizar a leitura dos dados de temperatura armazenados no Google Sheets, verificar anomalias nos valores e emitir avisos ao usuário caso a temperatura estivesse próxima ao limite da faixa desejada, ou fora dela.

Para a equipe de hardware, o resultado final era realizar a leitura da temperatura no interior e no exterior da geladeira, verificar se a porta estava aberta ou fechada, emitir o aviso sonoro no caso de a porta ficar aberta por muito tempo e enviar essas informações para o Google Sheets.

Durante o desenvolvimento das estruturas de software e hardware foram observadas possibilidades de melhorias como, por exemplo, na conectividade da rede WiFi, permitindo sua reconfiguração pelo usuário em caso de mudança de rede.

Após cada equipe concluir suas tarefas, conforme descrito acima, foi realizada a junção dos resultados e obtida a primeira versão do produto final.

Vale destacar que os trabalhos do grupo de hardware começaram com as ligações dos componentes (microcontrolador e periféricos) utilizando uma Protoboard (Figura 3-a). Após a certificação da montagem e funcionalidade dos componentes, optou-se por migrar a montagem para uma placa de circuito impresso padrão (universal), onde foram soldados os componentes e realizadas as trilhas de interconexões (Figura 3-b). Foi então concebido o primeiro protótipo do projeto.

A partir do primeiro protótipo e das falhas que este apresentou, em especial em relação às conexões dos periféricos com a placa universal, foi concebido por todo o grupo de estudantes o design de uma placa de circuito impresso (PCB). Para essa etapa foi utilizando o software EasyEDA, disponível gratuitamente na internet. O resultado é apresentado na Figura 3-c. Antes da construção da PCB os estudantes, juntamente com os professores, definiram os conectores comerciais para o acoplamento entre placa e componentes. 
Figura 3 - Etapas das conexões desenvolvidas: a) Protoboard; b) Placa impressa universal;

c) PCB

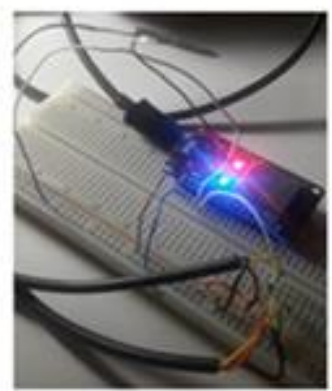

a)

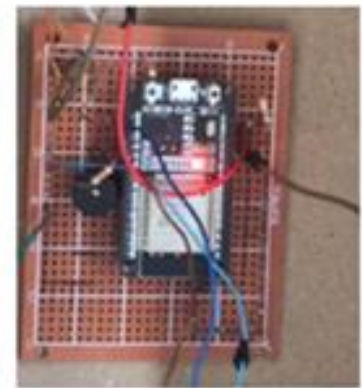

b)

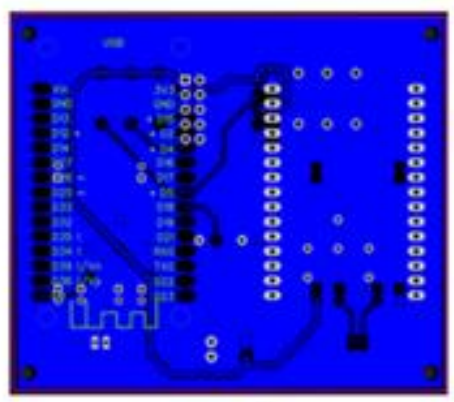

c)

Fonte: Os Autores.

\subsection{Avaliação do trabalho realizado}

Seguindo as etapas apresentadas anteriormente, chegou-se ao primeiro protótipo (Figura 3-b), com o qual foi possível realizar a medida de temperatura de geladeiras domésticas. Com o protótipo também foi possível analisar suas falhas e perceber melhorias, como por exemplo o aprimoramento de conexões na parte de hardware (Figura 3-c) e pequenos ajustes estéticos no aplicativo. Na seção abaixo (resultados) são apresentados mais detalhes sobre o produto final obtido com o desenvolvimento do projeto, além das etapas seguidas para sua obtenção.

\section{RESULTADOS}

Nesta seção serão discutidas e apresentadas as etapas e o produto final do projeto desenvolvido. O primeiro passo a ser apresentado é o levantamento dos requisitos e as opções escolhidas para a confecção do protótipo. Posteriormente, será detalhado o funcionamento do sistema em geral, com a descrição das características e funcionalidades do hardware e software.

\subsection{Levantamento de requisitos}

Tendo em vista a problemática relacionada aos métodos de mantenimento e armazenamento das vacinas na rede pública de saúde, o primeiro passo dado para a execução do projeto foi o levantamento de requisitos do sistema, ou seja, quais atividades e funções o protótipo realizaria e quais os melhores meios de se executar essas tarefas.

As principais funções do sistema e que foram levantadas no início do projeto foram: monitorar a temperatura interna de uma geladeira doméstica; alertar o estado da porta da geladeira (aberta ou fechada); garantir funcionamento do sistema caso ocorra queda de energia na rede elétrica; armazenar os dados de temperatura; monitoramento de dados via Web e App.

Para atender esses requisitos, um conjunto de indagações foi elaborado a fim de se pensar nas melhores opções de implementação do dispositivo. Os requisitos foram divididos em duas partes: software e hardware. O conjunto de indagações para a parte de software foram:

- Como o sistema irá se comunicar com a internet?

- Qual sistema de armazenamento na nuvem pode ser utilizado e facilitará a implementação do sistema? 
- Como mandar essas informações de temperatura para o app? Ou será feita através da internet e depois consultada pelo app?

- Como fazer a configuração da rede WiFi no sistema pela primeira vez?

- Como se comunicar no caso de falta de energia? Como garantir o WiFi?

- Como realizar o aviso de falta de energia para o app?

Para cumprir os requisitos relacionados ao hardware foram levantados os seguintes direcionamentos:

- Qual a melhor placa de microcontrolador para essa tarefa? E porquê?

- Qual o melhor sensor de porta aberta?

- Qual o melhor sensor de temperatura?

- Vai precisar de bateria? Qual tipo? E que circuitos que vou precisar para carregar?

- Como monitorar queda da rede elétrica?

- Como manter o dispositivo monitorando informações pelo maior tempo possível sem a rede elétrica?

- Qual deve ser a taxa de aquisição de dados?

Nas próximas seções serão abordadas as escolhas e as funções já desenvolvidas pelo sistema embarcado.

\subsection{Overview do sistema}

O sistema é composto por duas partes principais, hardware e software. O hardware é responsável por captar a temperatura, verificar se a porta está aberta ou fechada e ativar um alarme caso a porta permaneça aberta por um tempo considerado excessivo. Já o software implementa a aplicação mobile, responsável por armazenar as informações enviadas pelo hardware, informar ao usuário caso a temperatura saia da faixa definida, ou esteja próxima aos limites desejados, através de notificações no aplicativo. A comunicação entre essas partes é realizada através de uma planilha e uma API (Application Programming Interface) do Google Drive. Um diagrama representando o sistema é apresentado na Figura 4.

Figura 4 - Diagrama do sistema

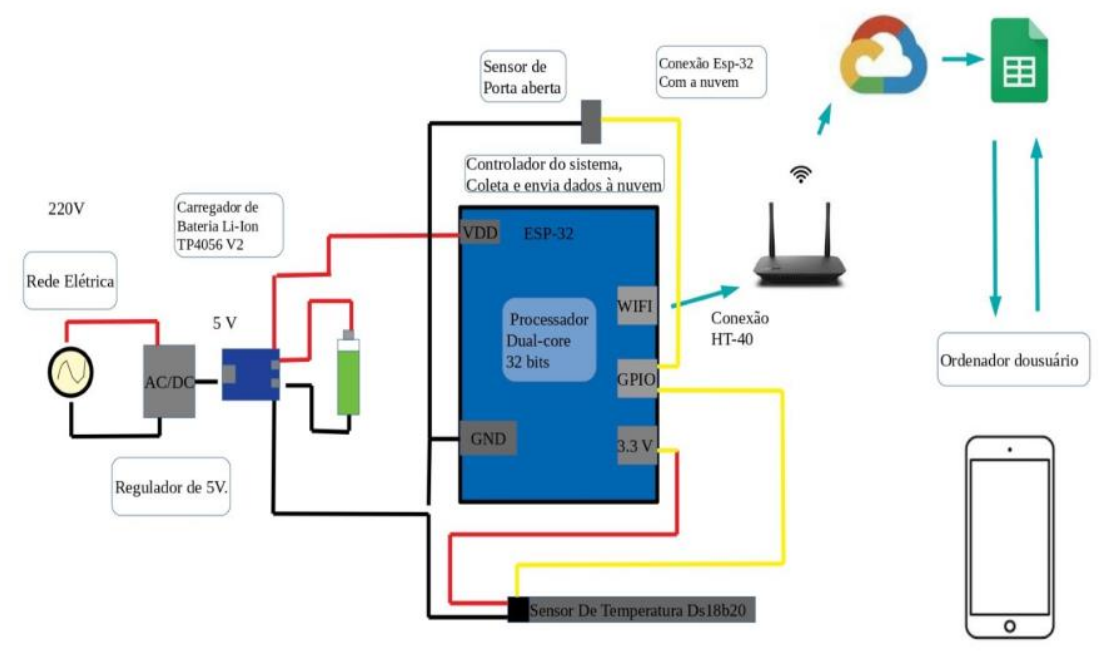

Fonte: Os Autores.

\subsection{Software}


O software foi desenvolvido na IDE (Integrated Development Environment) do Android Studio, um programa de computador que reúne características e ferramentas de apoio ao desenvolvimento de software com o objetivo de agilizar este processo. A linguagem de programação utilizada para o desenvolvimento é o Java e o código é estruturado em classes e separado em arquivos de interface gráfica e de estrutura e tarefas de cada página da aplicação.

A aplicação foi dividida em 5 páginas. Logo, ao instalar a aplicação e abri-la, a primeira página a aparecer é a de login, na qual o usuário registra-se através de uma conta Google. Após a conexão, com o registro realizado, a próxima página recebe o código de segurança necessário para conectar o aplicativo com o respectivo hardware. As próximas três páginas são de navegação no aplicativo. Com a conta e o código no aplicativo, esse segue para sua página principal, onde estão as informações de temperatura máxima e mínima do dia, temperatura atual, hora da última coleta de dados, além da temperatura externa. Nessa mesma página estão dois botões, um que leva para a página de perfil, na qual aparecem as informações da conta logada e a opção de sair dessa conta, e outro que leva para a página de atualização das informações. Essa página acessa a planilha para qual o hardware envia as informações e gera um gráfico com as últimas informações de temperatura interna.

\subsection{Hardware}

Nesta seção serão listados todos os componentes que compõe o funcionamento do hardware e seus respectivos esquemas de montagem. Além da listagem, serão detalhadas as funções de cada componente para aplicação do sistema.

\section{Nodemcu Esp32}

O módulo ESP32 é um módulo de alta performance para aplicações envolvendo WiFi (Wireless Fidelity), contando com um baixo consumo de energia e custo. As funções realizadas pelo ESP32 serão descritas a seguir:

- Conexão WiFi: O ESP32 utiliza o Wifi para enviar os dados para a planilha, os quais posteriormente serão lidas pelo aplicativo.

- Aquisição de dados: Os sensores de temperatura enviam os dados para a planilha. Porém, para seu funcionamento, são necessárias a alimentação e uma porta para armazenamento destes dados. O ESP32 realiza tais funções.

- Controle de porta aberta: Uma porta do ESP32 verifica o envio de sinal alto ou baixo, para porta aberta ou fechada.

- Avisos: Caso a porta permaneça aberta por um tempo prolongado, um aviso sonoro será enviado por um buzzer controlado pelo ESP32.

Para o funcionamento ininterrupto foi integrado ao protótipo um circuito de alimentação com Módulo TP4056. Ele mantém carregada uma bateria de lítio que é utilizada como fonte de alimentação em casos de falta de energia na rede elétrica local (Figura 4).

\section{Sensor de temperatura Ds18b20}

O sensor de temperatura utilizado para monitorar o interior da geladeira convencional e a temperatura externa foi o modelo DS18B20. Este sensor é a prova de água e conta com uma interface digital capaz de fornecer a leitura da temperatura através de uma única linha de comunicação com uma precisão de 12 bits e exatidão de $\pm 0.5^{\circ} \mathrm{C}$ 
Este módulo utiliza o circuito integrado TP4056, o qual é um carregador linear com corrente/tensão constante de uma célula de lítio, conta com led indicadore de carga e conexão mini-usb.

\section{Outros}

Uma bateria de 3,6V 9800mAh é responsável para manter a energia do dispositivo caso falte energia na rede elétrica local. Também foi utilizado um buzzer que serve como alarme sonoro caso a porta esteja em um estado indesejado.

\subsection{Sistema embarcado}

Com a união dos resultados da parte de software e hardware o projeto chegou à primeira fase de testes. Nessa etapa dois sensores de temperatura foram utilizados, um para o interior e outro para o exterior da geladeira convencional. Os dados coletados pelos sensores foram enviados para uma planilha do Google Sheets.

A partir dessas informações o aplicativo de celular mostra na página principal os seguintes dados (Figura 5-a): a temperatura interna máxima e mínima do dia, a temperatura interna da última coleta, a hora da última coleta e a temperatura externa durante a última coleta. Também há a possibilidade de gerar um gráfico a partir dos dados de temperatura interna obtidos, como mostra a Figura 5-b.

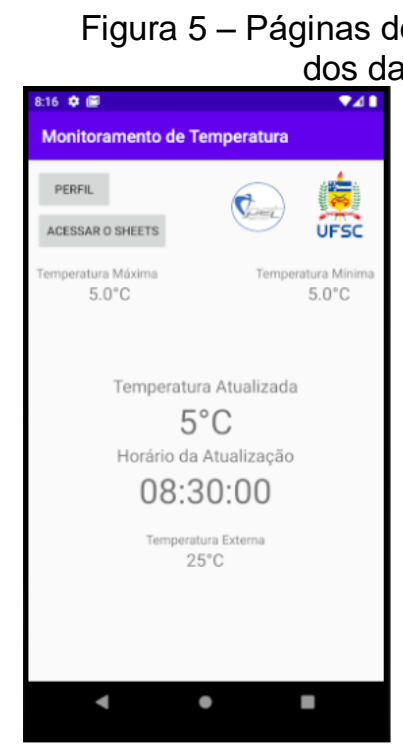

a)

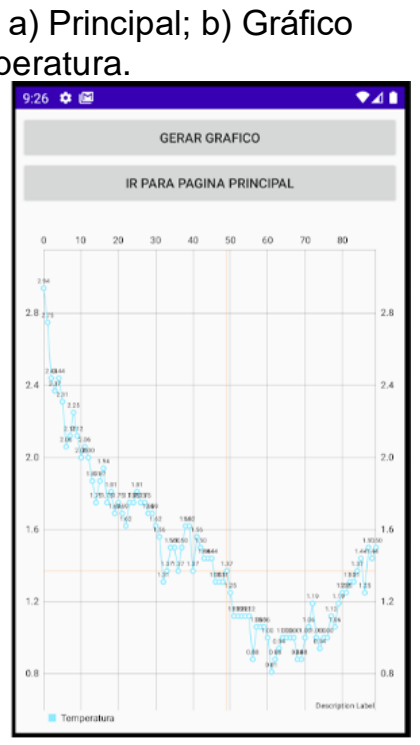

b)

Fonte: Os autores.

\section{$4 \quad$ CONCLUSÕES}

Em relação à proposta de aprendizagem por projetos, os estudantes destacam que a participação no processo ofereceu a eles a possibilidade de implementar de forma prática muitos tópicos que são abordadas nas ementas das disciplinas do curso de engenharia, como: montagem de um circuito utilizando componentes como resistores e capacitores; implementação de sensores e análise dos tipos disponíveis para cada aplicação; interpretação de datasheet de componentes; programação básica de microcontroladores; trabalho com dados através de software e hardware; desenvolvimento de aplicações de 
celular em linguagem Java; realizar a comunicação entre uma aplicação mobile e um banco de dados da nuvem utilizando ferramentas do Google; habilidades de design de PCB.

Os estudantes ressaltam ainda que o desenvolvimento do projeto também permitiu aprender a trabalhar de modo remoto e em equipe, o que lhes exigiu organização e responsabilidade no cumprimento de tarefas e prazos, algo que consideram muito importante para sua futura carreira profissional.

Em relação ao protótipo desenvolvido, esse permite o monitoramento da temperatura de uma geladeira doméstica utilizada para armazenamento de vacinas na rede pública de saúde. O equipamento também emite avisos, através de um aplicativo para celular, sobre eventuais faltas de energia na rede elétrica local e quando a temperatura está próxima de seus valores máximo e mínimo permitidos.

Por fim, após a conclusão da PCB será dado início aos testes do equipamento de forma mais efetiva, buscando implementá-lo nas unidades básicas de saúde da região de Joinville.

\section{Agradecimentos}

Os autores agradecem o Ministério da Educação e ao FNDE pela oportunidade de bolsa dentro do Programa de Educação Tutorial das Engenharias da Mobilidade (PET EMB). À universidade pelo apoio ao programa e apoio ao nosso projeto. Ao nosso tutor pelo suporte em todo projeto, e o suporte e trabalho conjunto dos colegas de equipe.

\section{REFERÊNCIAS}

Da Silva Santos JA, Torres DB, de Melo RA, Fernandes FECV, de Lacerda Campos MEA. Fatores determinantes das perdas das vacinas em Unidades Básicas de Saúde. Id On Line Revista Multidisciplinar E De Psicologia, 2016; 10(32):41-53.

FELIZONA, A. C. Rede de Frio: entenda o controle de qualidade das vacinas em todo o país. Disponível em: http://www.blog.saude.gov.br/index.php/entenda-o-sus/52532-redede-frio-uma-logistica-para-o-controle-de-qualidade-das-vacinas-em-todo-o-pais. Acesso em: 27 mar. 2019.

FIOCRUZ. Quais doenças foram erradicadas pela vacinação? Disponível em: https://www.bio.fiocruz.br/index.php/br/perguntas-frequentes/69-perguntasfrequentes/perguntas-frequentes-vacinas/221-quais-doencas-foram-erradicadas-pelavacinacao. Acesso em: 29 mar. 2019.

FONSECA, Sandra Medeiros, MATTAR, Joao. Metodologias ativas aplicadas à educação a distância: revisão da literatura. Revista EDaPECI, 2017; v. 17, n. 2, p. 185197.

FORBIDDEN. Por que vacinas são importantes? Disponível em: http://brasil.planetasaber.com/theworld/. Acesso em: 26 mar. 2018.

INDREL. INDREL Scientific - Industria de Refrigeração Científica de Londrina. Disponível em: https://www.indrel.com.br/. Acesso em: 06 ago. 2019.

L. Mikhailov, S. Mikhailova, R. Yersaiyn, G. Ismailova, N. Kenes, and R. Makhmutov. Modernization of mechatronic smart windows system to counteract the spread of COVID- 
19. Journal of Physics: Conference Series, aug. 2020. Disponível em: https://doi.org/10.1088/1742-6596/1615/1/012018. Acesso em: 01 abr. 2021.

MINISTÉRIO DA SAÚDE. Manual de Rede de Frio. 27 mar, 2019.

PET EMB. PET Engenharia da Mobilidade. Disponível em: https://petemb.ufsc.br/. Acesso em: 04 abr. 2021.

PORVIR. Aprendizagem baseada em projetos. Disponível em: https://porvir.org/aprendizagem-baseada-em-projetos/. Acesso em: 04 abr. 2021.

ROCHA, G. Programa Nacional de Imunizações (PNI). Disponível em: http://www.blog.saude.gov.br/index.php/entenda-o-sus/50027-programa-nacional-deimunizacoes-pni. Acesso em: 27 mar. 2019.

TUNÁPOLIS, Custo FECAM. Refrigeração Médica. Disponível em: https://static.fecam.net.br/uploads/452/arquivos/874182 BIOTEC00.pdf. Acesso em: 27 ago. 2019.

W. H. Organization. Coronavirus disease (covid-19). Disponível em: https://www.who.int/emergencies/diseases/novel-coronavirus-2019/question-and-answershub/q-a-detail/coronavirus-disease-covid-

19\%22\%20//\%20\%22: :text=symptoms\%22\%20/n\%20_blan Acesso em: 20 mar. 2021.

W. H. Organization. Coronavirus disease (covid-19) dashboard. Disponível em: https://covid19.who.int/. Acesso em: 20 mar. 2021.

\title{
EMBEDDED SYSTEM FOR VACCINE MONITORING: PROJECT BASED LEARNING IN THE PET EMB
}

\begin{abstract}
This paper presents the experience report of students in the Tutorial Education Program from the Mobility Engineering Center at UFSC, during the COVID-19 pandemic. The students developed a low-cost device for monitoring the temperature of domestic refrigerators used to store vaccines. The adopted methodology was the methodology based on projects, which outcome was the acquisition of teamwork skills in remote mode and the knowledge of low-cost embedded systems design. Additionally, the project results in the construction of an embedded system prototype composed by an application for mobile devices, which issues warnings in the event of a power outage and temperatures close to dangerous levels; and an embedded hardware, which acquires and sends the temperature data via the internet to a Google Sheets ${ }^{T M}$ spreadsheet.
\end{abstract}

Keywords: Vaccine. Monitoring. Mobile 